\title{
Fos-expressing neuronal ensemble in rat ventromedial prefrontal cortex encodes cocaine seeking but not food seeking in rats
}

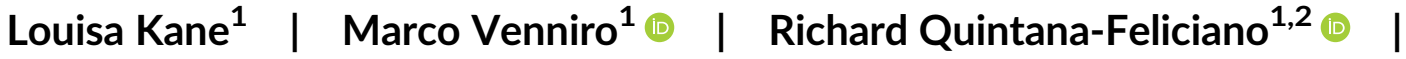 \\ Rajtarun Madangopal $^{1}$ (c) | F. Javier Rubio ${ }^{1}$ | Jennifer M. Bossert ${ }^{1}{ }^{\oplus}$ | \\ Daniele Caprioli $^{3,4}$ | Yavin Shaham ${ }^{1}$ (1) | Bruce T. Hope ${ }^{1}$ () | Brandon L. Warren ${ }^{1,2}$ (c)
}

\author{
${ }^{1}$ Behavioral Neuroscience Branch, \\ IRP/NIDA/NIH/DHHS, Baltimore, Maryland, \\ USA \\ ${ }^{2}$ Department of Pharmacodynamics, \\ University of Florida, Gainesville, Florida, USA \\ ${ }^{3}$ Santa Lucia Foundation (IRCCS Fondazione \\ Santa Lucia), Rome, Italy \\ ${ }^{4}$ Department of Physiology and Pharmacology, \\ Sapienza University of Rome, Rome, Italy

\section{Correspondence} \\ Dr. Brandon Warren, Department of \\ Pharmacodynamics, University of Florida, \\ Gainesville, FL 32610, USA. \\ Email: brandon.warren@ufl.edu \\ Funding information \\ National Institutes of Health, Grant/Award \\ Numbers: DA042102, Intramural Research \\ Program; Brain and Behavior Research \\ Foundation, Grant/Award Number: Young \\ Investigator Grant; National Institute on Drug \\ Abuse (NIDA), Grant/Award Number: \\ 4R00DA042102-02; National Institute on \\ Drug Abuse, Intramural Research Program, NIH
}

\begin{abstract}
Neuronal ensembles in ventromedial prefrontal cortex (VmPFC) play a role in both cocaine and palatable food seeking. However, it is unknown whether similar or different vmPFC neuronal ensembles mediate food and cocaine seeking. Here, we used the Daun02 inactivation procedure to assess whether the neuronal ensembles mediating food and cocaine seeking can be functionally distinguished. We trained male and female Fos-LacZ rats to self-administer palatable food pellets and cocaine on alternating days for 18 days. We then exposed the rats to a brief nonreinforced food- or cocaine-seeking test to induce Fos and $\beta$-gal in neuronal ensembles associated with food or cocaine seeking, respectively and infused Daun02 into vmPFC to ablate the $\beta$-gal-expressing ensembles. Two days later, we tested the rats for food or cocaine seeking under extinction conditions. Although inactivation of the foodseeking ensemble did not influence food or cocaine seeking, inactivation of the cocaine-seeking ensemble reduced cocaine seeking but not food seeking. Results indicate that the neuronal ensemble activated by cocaine seeking in vmPFC is functionally separate from the ensemble activated by food seeking.
\end{abstract}

\section{KEYWORDS}

addiction, Daun02 inactivation, operant conditioning, vmPFC

\section{1 | INTRODUCTION}

A key feature of drug addiction is that in some individuals, drug taking persists despite the loss of alternative nondrug social, occupational, or recreational rewards. ${ }^{1-3}$ Individuals with cocaine use disorder report increased craving in response to cocaine-associated cues but not to other unrelated stimuli, suggesting a high degree of cue specificity. ${ }^{4,5}$

The prefrontal cortex is activated by exposure to cocaine and cocaine-associated cues in both human drug users ${ }^{6,7}$ and in preclinical studies. $^{8,9}$ Following cocaine exposure, medial prefrontal cortex (mPFC) neurons increase dendritic spines. ${ }^{10,11}$ Reversal of cocaineinduced synaptic changes in mPFC by inhibition of the $\beta$-adrenergic receptor signaling impairs recall of contexts previously paired with cocaine. ${ }^{12,13}$ However, it is unlikely that these types of nonspecific global changes are responsible for the highly specific cocaine-related learning that drives cocaine seeking. These learned associations are likely encoded within patterns of sparsely distributed neurons, termed "neuronal ensembles," that are strongly activated by cues and reinforcers and can be identified by the expression of the immediate early gene Fos, a marker of strong neuronal activation. ${ }^{14-17}$

Until relatively recently, the tools required to test the role of neuronal ensembles in drug-seeking have not been available. Recent reports indicate that neuronal ensembles are critical for conditioned responses and relapse to several drugs, including cocaine, heroin, 
alcohol, nicotine, and methamphetamine. ${ }^{18-26}$ In a previous studies, we found that neuronal ensembles within ventral mPFC are critical for both cocaine and food seeking. ${ }^{24,27}$ We also found that neuronal ensembles within dorsomedial striatum are critical for methamphetamine seeking but not food seeking. ${ }^{19}$ These studies suggest that distinct neuronal ensembles mediate drug seeking versus food seeking. Here, we build on these findings and tested the hypothesis that distinct nonoverlapping neuronal ensembles within ventral mPFC control either palatable food or cocaine seeking.

For this purpose, we used Fos-LacZ transgenic rats and the Daun02 inactivation procedure ${ }^{16,28}$ to ablate ventral mPFC ensembles reactivated during re-exposure to palatable food- or cocaineassociated cues and subsequently tested the effect of this ablation on food and cocaine seeking. We hypothesized that the ablation of cocaine-associated ensemble will selectively decrease cocaine but not food seeking and vice versa.

\section{2 | MATERIALS AND METHODS}

\section{1 | Subjects}

We used male Sprague Dawley rats $(n=24)$ in experiment 1 and both male and female Fos-lac $Z$ transgenic rats $(n=64)$ in experiments 2 and 3 , weighing $175-400 \mathrm{~g}$ at the beginning of the experiments. Fos-lac $Z$ transgenic rats were previously bred for 45-50 generations on a Sprague Dawley background. We housed rats (two per cage) by sex for at least 1 week before surgery and individually housed rats after intravenous surgery. We maintained the rats on a reverse 12-h light/dark cycle (lights off at 8:00 a.m.). We food-restricted rats to $20 \mathrm{~g} /$ day of standard rat chow beginning 2 days before starting operant training. Once rats self-administered both palatable food pellets and cocaine, we fed them ad libitum. Our procedures followed the guidelines outlined in the National Institutes of Health Guide for the Care and Use of Laboratory Animals (http://grants.nih.gov/grants/olaw/ Guide-for-the-Care-and-Use-of-Laboratory-Animals.pdf). We excluded one Sprague Dawley and five Fos-lacZ transgenic rats because of loss of catheter patency or misplaced cannulas.

\section{2 | Surgery}

We anesthetized rats with isoflurane (5\% induction, $3 \%$ maintenance, Butler Schein) and injected ketoprofen $(2.5 \mathrm{mg} / \mathrm{kg}$, s.c., Butler Schein) for 3 days after surgery to relieve pain and reduce inflammation. We allowed rats to recover from surgery for a minimum of 3-7 days.

\subsection{1 | Intravenous catheter implantation}

We implanted silastic catheters into the jugular vein. ${ }^{19,20,29}$ We placed the distal end of the catheter into the jugular vein and attached the proximal end of the catheter to a modified 22-gauge cannula. We ported the cannula through the midscapular region of the back. We flushed the catheters daily with gentamicin in sterile saline $(4.25 \mathrm{mg} /$ $\mathrm{ml}$; APP Pharmaceuticals).

\subsection{2 | Intracranial cannula implantation}

We implanted permanent guide cannulas (23-gauge, Plastics One) bilaterally $1 \mathrm{~mm}$ above ventromedial prefrontal cortex (vmPFC). The nose bar was set at $-3.3 \mathrm{~mm}$, and the coordinates for VmPFC were anteroposterior, +3.0 ; mediolateral, \pm 1.5 ; and dorsoventral, $-3.8\left(10^{\circ}\right.$ angle). We fixed cannulas to the rat's skull with dental cement and jeweler's screws. We used the above coordinates based on pilot and previous studies. $^{18,27}$

\section{3 | Intracranial injections}

We performed intracranial injections using a syringe pump (Harvard Apparatus) and $10-\mu \mathrm{l}$ Hamilton syringes attached via polyethylene-50 tubing to 30-gauge injectors (Plastics One) that extended $1 \mathrm{~mm}$ beyond the guide cannula. We infused $0.5 \mu$ over $1 \mathrm{~min}$ and left the injectors in place for one more minute before removal.

\section{4 | Drugs}

We received cocaine $\mathrm{HCl}$ (cocaine) from NIDA pharmacy. We chose a self-administration dose of $1.0 \mathrm{mg} / \mathrm{kg}$ per infusion based on previous studies. ${ }^{20}$ In experiments 2 and 3, on induction day, we injected Daun02 or vehicle into the vmPFC. We obtained Daun02 from Sequoia Research Products (www.seqchem.com). We dissolved Daun02 ( $2 \mu \mathrm{g} / 0.5 \mu \mathrm{l}$ per side) in vehicle solution containing 5\% DMSO, 6\% Tween 80, and 89\% 0.01M phosphatebuffered saline (PBS). We chose the dose of Daun02 based on previous studies. ${ }^{18,20,28}$

\section{5 | Apparatus}

We trained and tested the rats in Med Associates self-administration chambers; each chamber was equipped with two operant panels on opposite sides of the chamber. We equipped the left panel with a discriminative stimulus (red light) that signaled the insertion and subsequent availability of the cocaine-paired active (retractable) lever and a white cue light. We equipped the right panel with a discriminative stimulus (white house light) that signaled the insertion and subsequent availability of the food-paired active (retractable) lever, a tone-cue generator, and food port connected to an automated pellet dispenser. We also equipped the right panel with an inactive (stationary) lever that had no programmed consequences. 


\section{6 | Tissue preparation and Fos immunohistochemistry}

Ninety minutes after the start of the test session, we anesthetized rats with isoflurane for $90 \mathrm{~s}$ and perfused them with $100 \mathrm{ml}$ of PBS, followed by $400 \mathrm{ml}$ of $4 \%$ paraformaldehyde in 0.1M PBS. We postfixed the brains for an additional 1-4 $\mathrm{h}$ in paraformaldehyde and cryoprotected them in $30 \%$ sucrose in PBS at $4^{\circ} \mathrm{C}$ for $2-3$ days. We froze the brains in dry ice and kept them at $-80^{\circ} \mathrm{C}$ until sectioning.

We washed coronal brain sections $(40 \mu \mathrm{m})$ in PBS, blocked with $3 \%$ normal goat serum (NGS) in PBS with $0.25 \%$ Triton X-100 (PBS$\mathrm{Tx}$ ), and incubated $24 \mathrm{~h}$ at $4{ }^{\circ} \mathrm{C}$ with anti-Fos antibody (1:5000 dilution; Cell Signaling Technology Cat\# 5348, RRID: AB_10557109) in blocking solution. We then washed sections in PBS and incubated them with biotinylated goat antirabbit secondary antibody (1:600 dilution; Vector Labs, BA-9400; RRID: AB_2336202) in PBS-Tx and 1\% NGS for $2 \mathrm{~h}$. After washing in PBS, we incubated sections for $1 \mathrm{~h}$ in avidin-biotin-peroxidase complex (ABC Elite kit, PK-6100; Vector Laboratories) in PBS containing 0.5\% Triton X-100.

Finally, we washed sections in PBS and developed in 3,3'diaminobenzidine for $\sim 3 \mathrm{~min}$, washed with PBS, and mounted onto chromalum gelatin-coated slides. Once dry, we dehydrated the slides through a graded series of alcohol (30\%, 60\%, 90\%, 100\%, $100 \%$ ethanol) and cleared them with Citrisolv (Fisher Scientific) before coverslipping with Permount (Sigma). We digitally captured bright-field images of immunoreactive cells in vmPFC using an EXi Aqua camera (QImaging) attached to a Zeiss Axioskop 2 microscope with 20x objective (Carl Zeiss Microscopy) and iVision software for Macintosh, version 4.0.15 (Biovision). Observers (BLW and LK) blind to the test conditions (interrater reliability: Pearson's $r=0.89$ ) automatically counted labeled nuclei from two sections (bilateral) per rat (three images per rat) using NIH ImageJ. We averaged the counts so that each rat was an $n$ of 1 for each brain area.

\section{7 | Self-administration training for food and cocaine rewards}

Each experiment consisted of 3 weeks of self-administration training for $3 \mathrm{~h}$ /day. Each 3-h session was divided into three 1-h sessions, with a 10-min off period between sessions. Every day, we presented the rats with either the cocaine active lever or the food active lever, with corresponding cues and rewards contingent upon active lever presses. We started all rats on day 1 with food selfadministration training, in which we trained the rats to lever press to receive five palatable food pellets ${ }^{30}$ with a maximum daily limit of 45 deliveries.

On day 2 , we trained the rats to lever press to receive intravenous infusions of cocaine ( $1 \mathrm{mg} / \mathrm{kg}$ per infusion) with a maximum daily limit of 45 infusions. Upon reaching the daily limit, the lever retracted and the house light turned off, ending the session. Rats earned infusions on a fixed ratio 1 with 20-s timeout. We continued to alternate between food and cocaine sessions every day. As an aside, on the seventh day of each week, we gave the rats a 3-h choice session in which rats had 20 discrete choice trials. Each trial began with the extension of both the food and cocaine active levers (and presentation of the reward-associated cues). ${ }^{30}$ The rats had $10 \mathrm{~min}$ to choose between food and cocaine, after which both levers retracted for the duration of the 10-min period. This discrete trial choice procedure is based on previous work of Ahmed and colleagues. ${ }^{31,32}$ Following the choice session, the rats resumed alternating days of food and cocaine self-administration. This cycle repeated three times (totaling 21 days), with the final day of training being a choice session. We then returned all rats to their home cages for 7 days of forced abstinence from both rewards.

\subsection{Daun02 inactivation following induction of Fos and $\beta$-galactosidase}

One week after the last training session, we exposed the rats to a brief (30 min) induction session to induce reward specific Fos expression. During this induction session, the rats had access to the cues, contexts, and lever associated with cocaine or food but did not receive the actual reward (extinction conditions). We waited $90 \mathrm{~min}$ after the session start for Fos and $\beta$-galactosidase ( $\beta$-gal) expression and then infused either vehicle or Daun02 bilaterally into the $v m P F C$ to inactivate neuronal ensembles associated with cocaine or food seeking. Two days later, we performed two consecutive food- and cocaine-seeking tests under extinction conditions (counterbalanced). We perfused these rats after the test to check cannula placements.

\subsection{1 | Experiment 1: Effect of food and cocaine seeking on Fos expression in vmPFC}

The goal of experiment 1 was to determine whether exposure to cues previously associated with cocaine or food self-administration would induce Fos expression in vmPFC. The experimental timeline is shown in Figure 1A. We first trained rats to self-administer food or cocaine. Seven days later, we exposed rats in the test groups to a short 30-min nonreinforced induction session in which the rats pressed the lever for the cue previously associated with food or cocaine delivery. We matched all groups based on lever pressing during the last day of self-administration training. We reasoned that for rats in the food recall group, the predominant memories recalled during the 30-min test session would be the food selfadministration memories, whereas for rats from the cocaine recall group, the predominant memories recalled would be the cocaine self-administration memories. We left the rats in the no-test group in their home cage on test day. Ninety minutes after the start of the test, we perfused the rats and extracted their brains for Fos immunohistochemistry. 


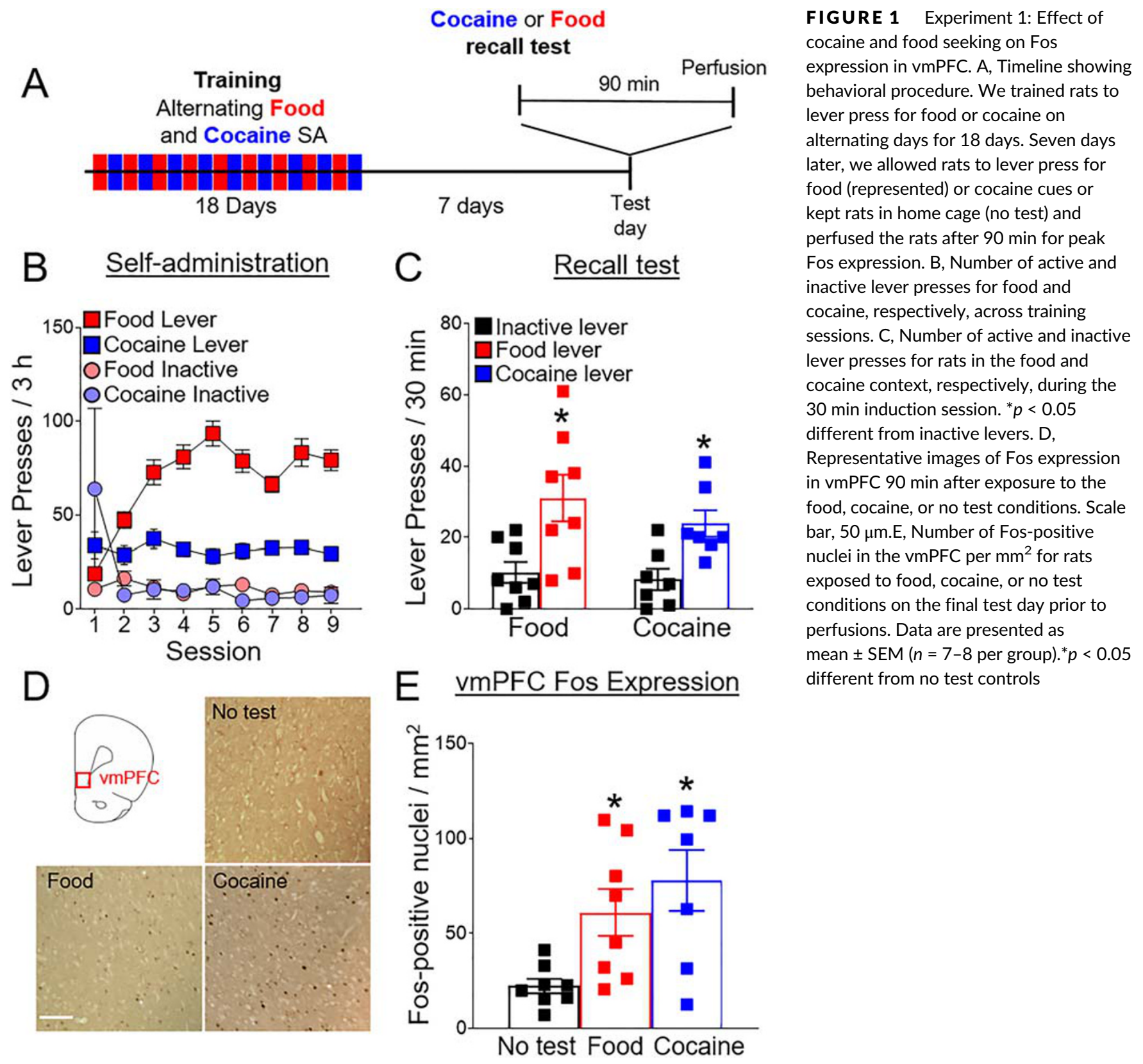

\subsection{2 | Experiment 2: Effect of Daun02 inactivation of neurons in vmPFC previously activated during food seeking on subsequent food and cocaine seeking}

We used the Daun02 inactivation procedure ${ }^{18,20,28}$ to determine whether Fos-expressing neuronal ensembles in vmPFC associated with food seeking play a causal role in the food or cocaine seeking. The experimental procedure was similar to that of experiment 1 , with the exception that we added a short 30-min induction session to reactivate the putative food self-administration ensemble. We hypothesized that food memories are recalled during induction day in rats exposed to food cues. Therefore, we predicted that postsession injections of Daun02 would inactivate ensembles encoding food selfadministration and impair food seeking in the test session 2 days later. We hypothesized that neuronal ensembles mediating food self- administration are selective for food self-administration. Therefore, we also tested rats' recall of cocaine self-administration in an identical test, $24 \mathrm{~h}$ later. We counterbalanced the order of the two tests so that some rats received the food-seeking test first, and some rats received the cocaine-seeking test first.

The experimental timeline is shown in Figure 2A. We anesthetized Fos-lac $Z$ rats and implanted permanent guide cannulas bilaterally targeting vmPFC. After a minimum of 7-day recovery, we trained rats using the same procedure described in experiment 1 . After training the rats to lever press for food or cocaine on alternating days for 18 days, we divided them into two groups that received vehicle or Daun02 on induction day.

On induction day, we placed the rats into the test chamber and allowed them to lever press for food-associated cues for $30 \mathrm{~min}$ to induce $\beta$-gal protein expression. We then returned rats to their home 


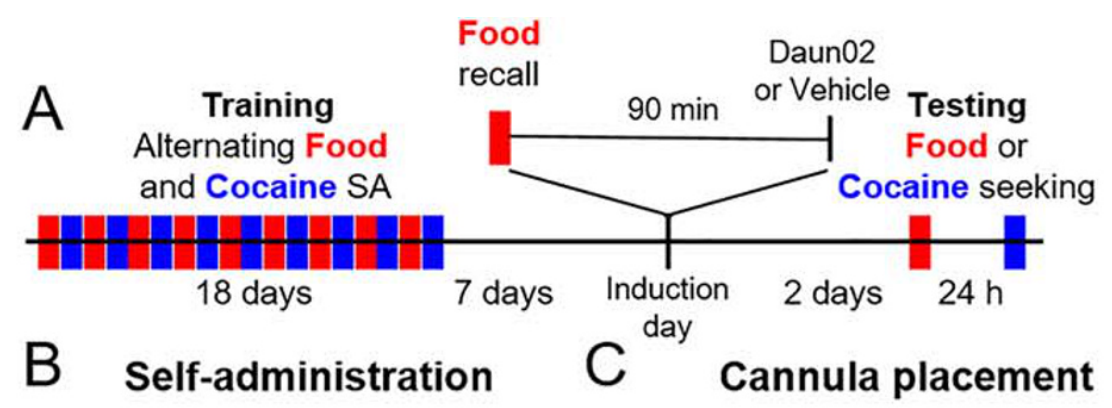

FIGURE 2 Experiment 2: Effect of food ensemble inactivation on food and cocaine seeking. A, Timeline showing behavioral procedure. We trained rats to lever press for food or cocaine on alternating days for 18 days. Seven days later, we allowed rats to lever press for food-associated cues and injected vehicle or Daun02 into vmPFC to inactivate the food ensemble. Two days later, we tested their ability to recall either the cocaine selfadministration memory or the food selfadministration memory. B, Number of active and inactive lever presses during self-administration of food and cocaine. C, Images showing placement of cannulas into vmPFC. D, Number of active and inactive lever presses over a 30-min extinction session on induction day. E, Number of active and inactive lever presses over a 30-min extinction session on test day. Data are presented as mean \pm SEM (vehicle, $n=14 ;$ Daun02, $n=14$ )
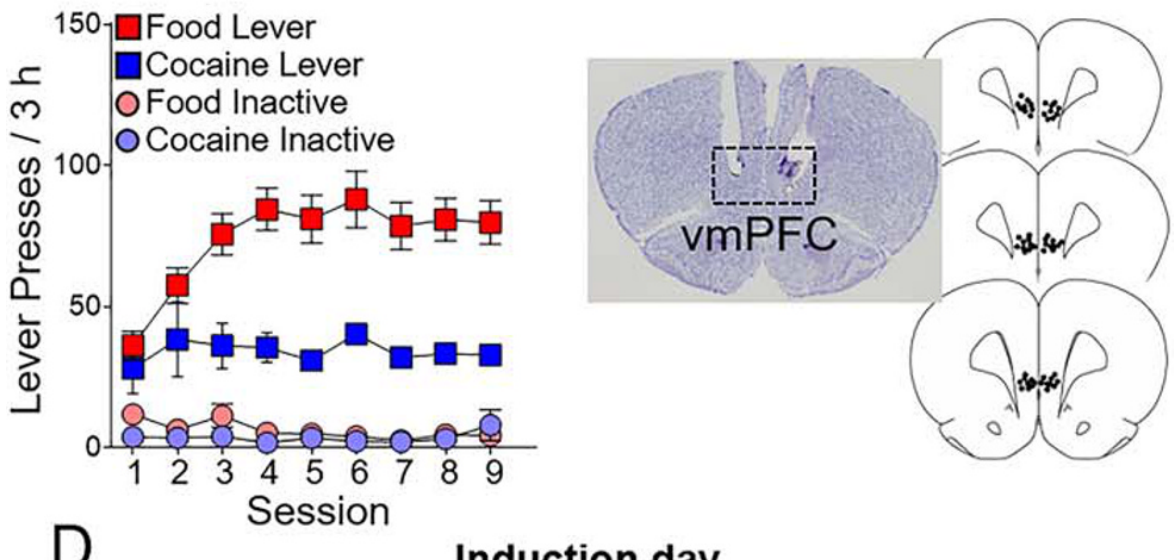

Induction day
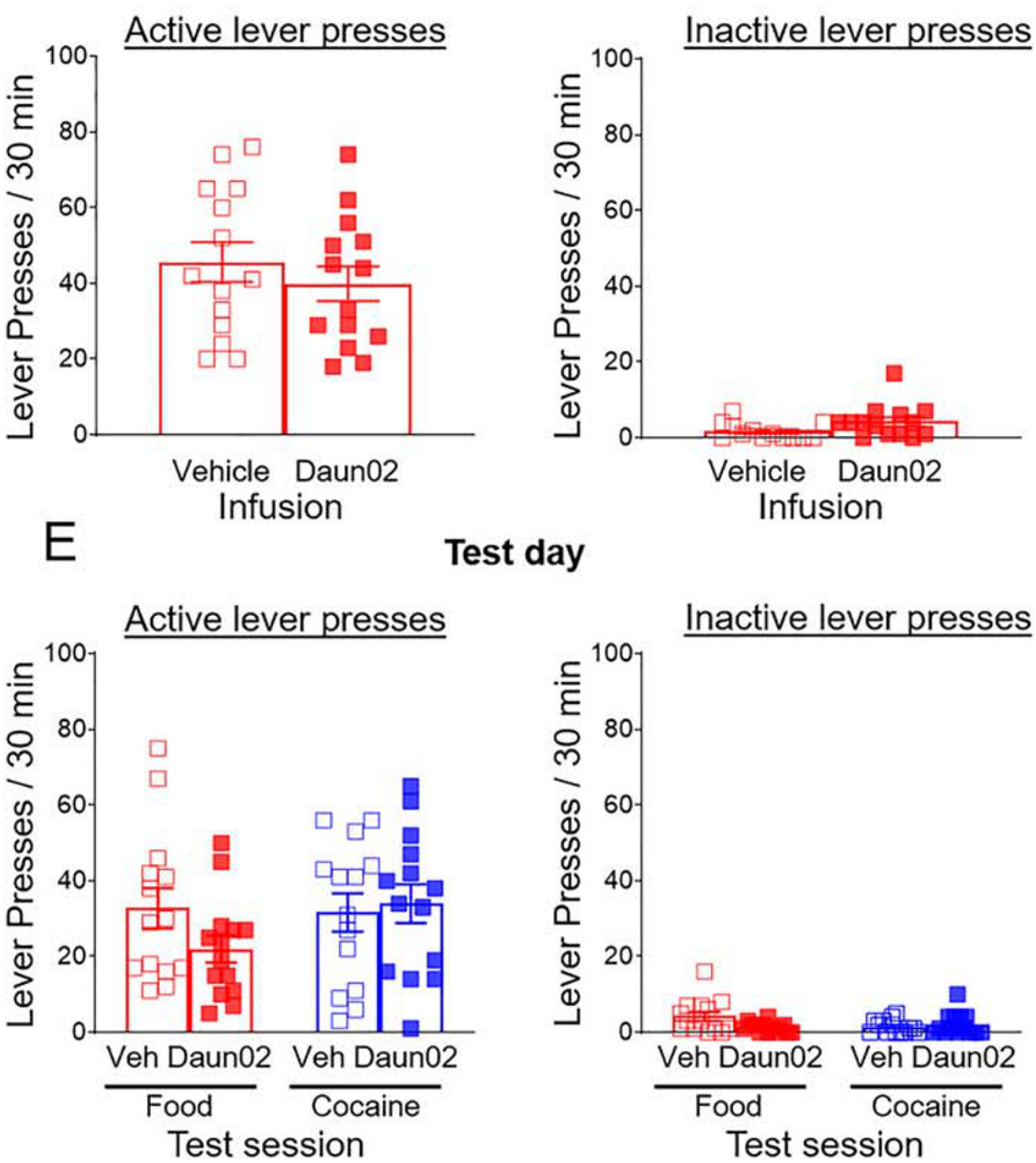

$\underline{\text { Inactive lever presses }}$

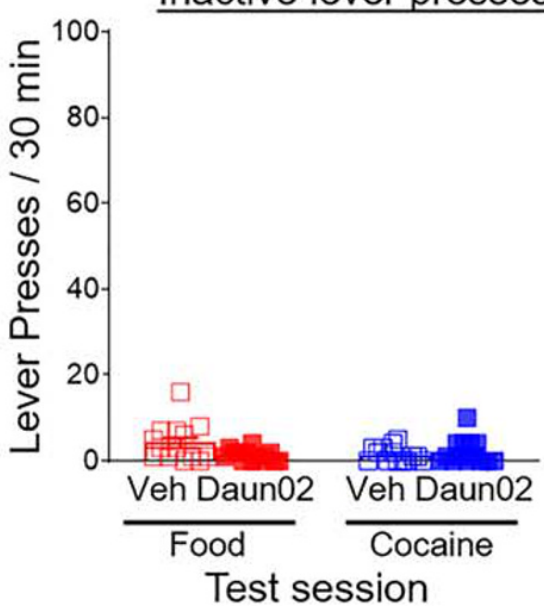


cages and, 90 min after the start of the induction session, injected vehicle or Daun02 into vmPFC. Two days later, we tested all rats for nonreinforced lever presses during a 30-min reward-seeking test. Following this test, we returned all rats to their home cages. Twenty-four hours later, we tested all rats in the alternate condition. Ninety minutes after the start of the last test, we perfused the rats and extracted their brains to check for cannula placements.

\subsection{3 | Experiment 3: Effect of Daun02 inactivation of neurons in vmPFC previously activated during cocaine seeking on subsequent cocaine and food seeking}

To test whether Fos-expressing neuronal ensembles in vmPFC associated with cocaine seeking play a causal role in the recall of food or cocaine self-administration, we performed a Daun02 inactivation experiment using Fos-LacZ transgenic rats $(n=31)$. This experiment is identical to experiment 2 except that we performed the inactivation in rats that had access to the cocaine lever and cues on induction day. The experimental timeline is shown in Figure 3A. We trained rats to self-administer food and cocaine as in experiments 1 and 2 . On induction day, 1 week after the last training session, we exposed the rats to a brief $(30 \mathrm{~min})$ session of cocaine self-administration recall. We waited 90 min for Fos and $\beta$-gal expression and then infused either vehicle or Daun02 into vmPFC to inactivate neuronal ensembles associated with cocaine seeking. Two days later, we exposed rats to a 30-min test of cocaine or food seeking. Twenty-four hours later, we tested all rats in the alternate condition. Ninety minutes after the start of the last test, we perfused the rats and extracted their brains to check for cannula placements.

\section{9 | Statistical analysis}

We analyzed the behavioral and immunohistochemistry data with Graphpad Prism 8 and SPSS. We used different analyses of variance (ANOVAs) based on the experimental design and the dependent measures to analyze the training, induction, and reward-seeking test data.

In experiment 1, we assessed Fos expression in vmPFC in 23 rats using a single factor between-subjects experimental design: Test (No test, Food recall, Cocaine recall). In experiment 2, we examined Fos-lacZ rats $(n=28)$ using $2 \times 2$ mixed factorial experimental design: Injection (vehicle, Daun02, between-subjects) $\times$ Reward type (food, cocaine, within-subjects), with inactive lever presses as a covariate. We included Reward type as a factor to assess whether Daun02 ablation of an ensemble affects one Reward type (food) and not the other (cocaine). Experiment 3 was a $2 \times 2$ mixed factorial experimental design: Injection (vehicle, Daun02, between-subjects) $\times$ Reward type (food, cocaine, within-subjects), including inactive lever presses as a covariate. We included Reward type as a factor to assess whether Daun02 ablation of an ensemble affects one Reward type (cocaine) and not the other (food).
In experiment 3, we identified two outliers using the explore command in SPSS and removed them from the analysis. We followed up on a significant main effect in experiment 1 and a significant interaction effect in experiments 2 and $3(p<0.05)$ with a Fisher PLSD posthoc test, a widely used post-hoc test when the number of post-hoc comparison is very low after a significant main effect in one-way ANOVA or a significant interaction in $2 \times 2$ factorial ANOVA. The data that support the findings of this study are available from the corresponding author upon reasonable request.

\section{3 | RESULTS}

\section{1 | Experiment 1: Effect of food and cocaine seeking on Fos expression in vmPFC}

We hypothesized that cocaine-specific versus food-specific cues would induce recall of food versus cocaine self-administration memories and their associated Fos-expressing ensembles in vmPFC.

\subsection{1 | Training}

Figure $1 \mathrm{~B}$ shows the mean \pm SEM number of active and inactive lever presses during the self-administration phase of experiment 1 . The rats showed reliable food and cocaine self-administration with higher responding for the food reward. The statistical analysis, which included the within-subjects factors of Reward type (cocaine, food), Lever (active, inactive), and training session (1-9), showed significant effects of Session $\times$ Reward type $\left(F_{8,352}=5.48, p<0.0001\right)$ and Session $\times$ Lever $\left(F_{8,352}=7.5, p<0.0001\right)$. During the weekly choice sessions (Figure S1-1A), the rats showed strong preference for the food reward $(p<0.01)$, in agreement with our previous studies with methamphetamine. $^{30}$

\subsection{2 | Reward-seeking test}

On the test day, we assessed nonreinforced lever pressing for $30 \mathrm{~min}$ (Figure $1 \mathrm{C}$ ). There were no differences between the cocaine and food conditions. The ANOVA, which included the betweensubjects factor of Reward type and Lever, showed a significant effect of Lever $\left(F_{1,26}=16.8, p=0.0004\right)$ but not Reward type or interaction $(p>0.1)$.

\subsection{3 | Fos}

We analyzed Fos expression in the vmPFC following the test recall sessions; sample images from vmPFC are shown in Figure 1D. There were no differences between the food and cocaine groups, and both groups were higher than the no-test group: Test day exposure (No test, Food recall, Cocaine recall), $F_{2,20}=6.1, p=0.008$. 


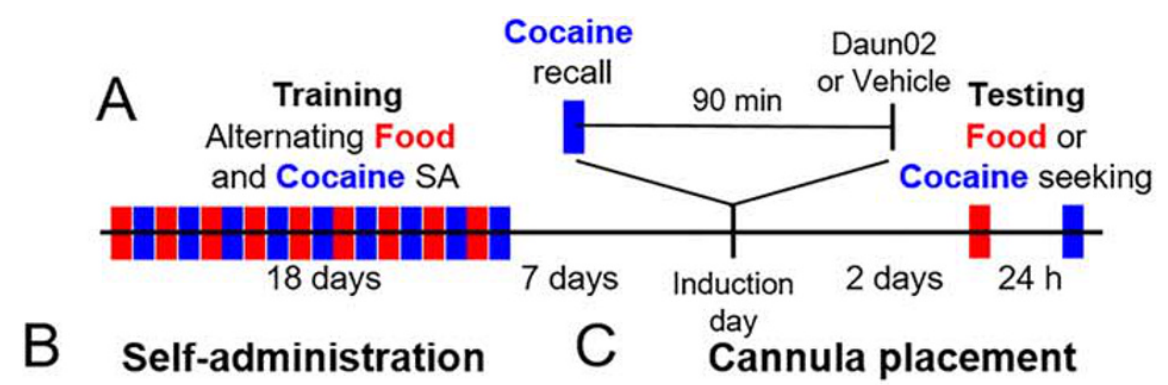

FIGURE 3 Experiment 3: Effect of cocaine ensemble inactivation on food and cocaine seeking. A, Timeline showing behavioral procedure. We trained rats to lever press for food or cocaine on alternating days for 18 days. Seven days later, we allowed rats to lever press for cocaine-associated cues and injected vehicle or Daun02 into vmPFC to inactivate the cocaine ensemble. Two days later, we tested their ability to recall either the cocaine self-administration memory or the food self-administration memory. B, Number of active and inactive lever presses during self-administration of food and cocaine. C, Images showing placement of cannulas into vmPFC. D, Number of active and inactive lever presses over a 30-min extinction session on induction day. E, Number of active and inactive lever presses over a 30-min extinction session on test day. Data are presented as mean \pm SEM (vehicle, $n=13$; Daun02, $n=16$ ). ${ }^{*} p<0.05$ different from vehicle for active lever presses
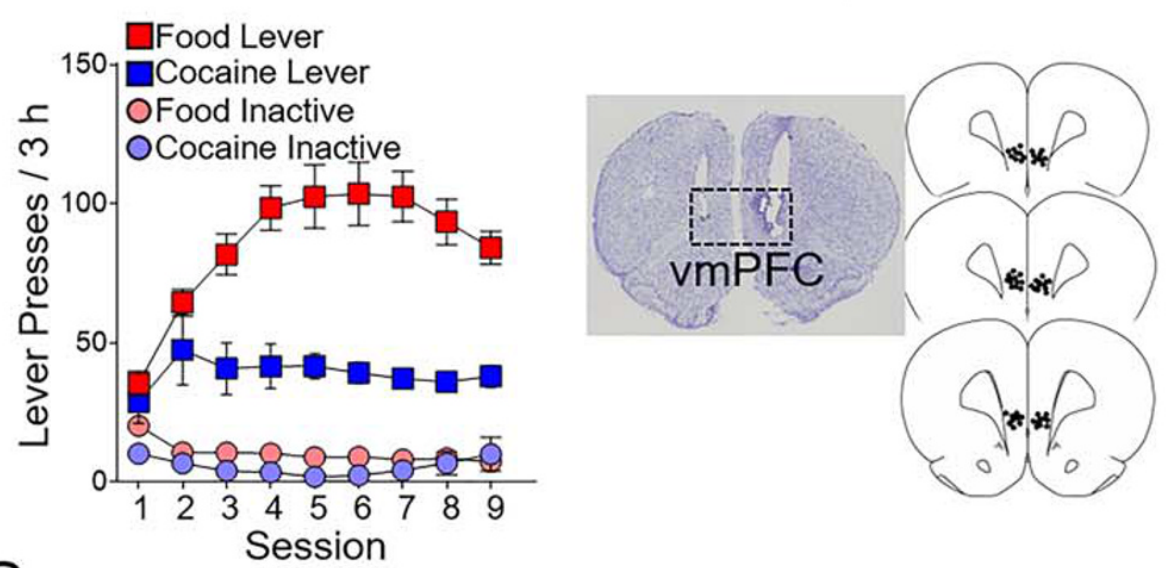

D

Induction day
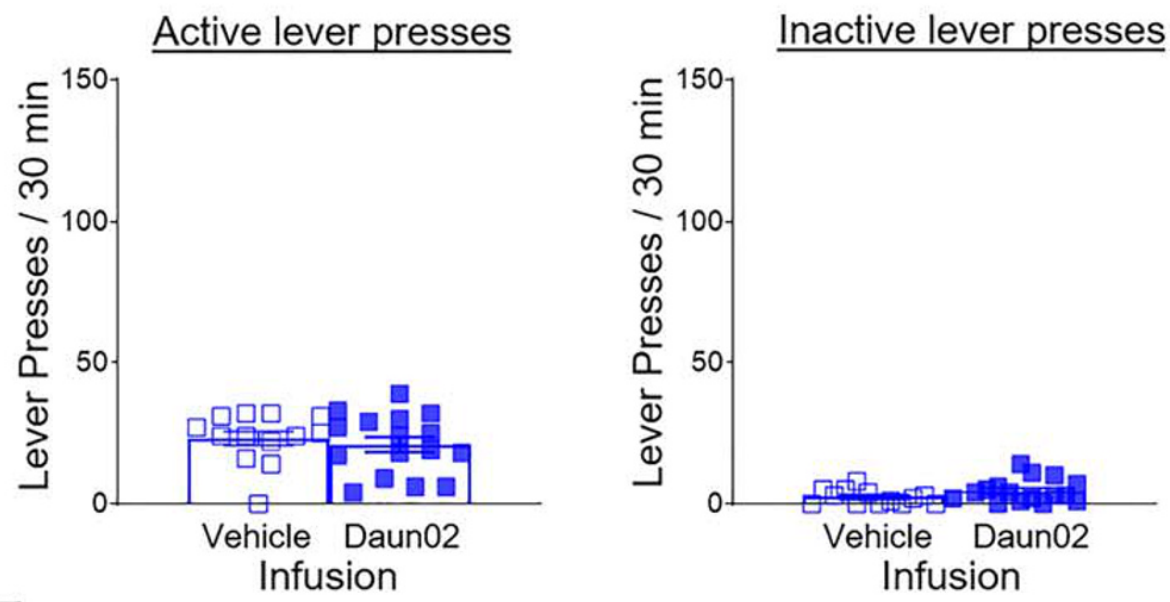

E

Test day

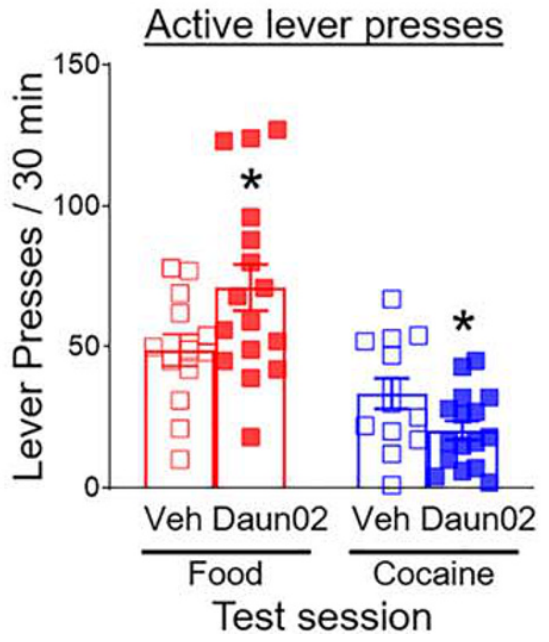

$\underline{\text { Inactive lever presses }}$

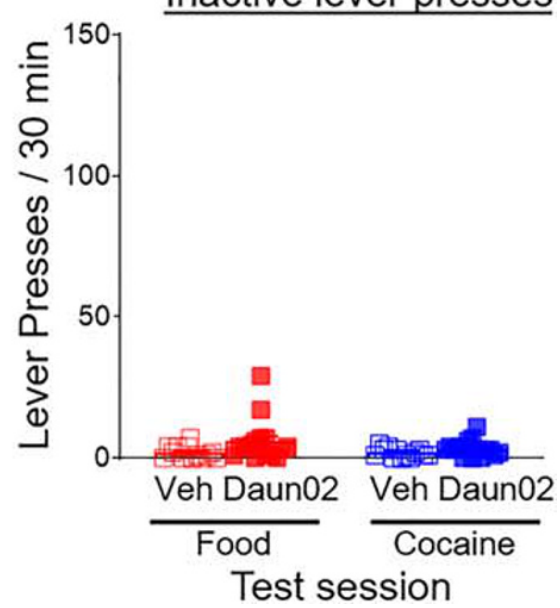




\subsection{Experiment 2: Effect of Daun02 inactivation of neurons in vmPFC activated during recall of the food self-administration memory on food and drug seeking}

We used the Daun02 inactivation procedure ${ }^{18,20,28}$ to determine whether Fos-expressing neuronal ensembles associated with food seeking in vmPFC play a causal role in food or cocaine seeking. For this purpose, we added a short 30-min induction session to activate the putative food self-administration ensemble and then ablate these neurons using postsession Daun02 injections. We tested whether ablating neuronal ensembles activated by food seeking during induction day would lead to decreases in food seeking without influencing cocaine seeking during the test day.

\subsection{1 | Training and induction day}

Figure $2 \mathrm{~A}$ shows the design of experiment 2 . Figure $2 \mathrm{~B}$ shows the mean \pm SEM number of active and inactive lever presses during the self-administration phase, and Figure S2-1B shows the results of the weekly choice sessions. As in experiment 1 , the rats showed reliable food and cocaine self-administration with higher responding for the food reward (Figure 2B) and also higher preference for the food reward during the choice sessions (Figure S2-1B; $p<0.01$ ).

\subsection{2 | Induction day (Figure 2D)}

On induction day, nonreinforced lever pressing for cues associated with food seeking was assessed for 30 min prior to being placed in their home cages for 60 min and injected with vehicle or Daun02 into vmPFC. There were no group differences in active lever presses prior to vehicle or Daun02 injections between the groups (Figure 2D; $p>0.1)$

\subsection{3 | Reward-seeking test (Figure 2E)}

We found no significant differences in behavioral responses between male and female rats on test day (Figure S2) and therefore combined these groups for analysis. Daun02 injections did not significantly decrease food or cocaine seeking during testing. The initial twofactorial mixed analysis of covariance (ANCOVA), which included the between-subjects factors of Daun02 condition (vehicle, Daun02), the within-subjects factor of Reward type (food, cocaine), and the covariate Inactive lever presses, showed a significant effect of Reward type $\left(F_{1,24}=4.8, p=0.04\right)$, but not Daun02 condition $\left(F_{1,24}=1.4, p=0.24\right)$, and no interaction between Daun02 condition $\times$ Reward type $\left(F_{1,24}=0.6, p=0.46\right)$. These results did not support our hypothesis that selective ablation of the putative food seeking ensemble on induction day would selectively decrease food seeking but not cocaine seeking on the test day.

\section{3 | Experiment 3: Effect of Daun02 inactivation of neurons in vmPFC activated during recall of the cocaine self-administration memory on food and cocaine seeking}

We used the Daun02 inactivation procedure to determine whether Fos-expressing neuronal ensembles associated with cocaine seeking in vmPFC play a causal role in food or cocaine seeking. Similar to experiment 2, we added a short 30-min induction session to activate the putative cocaine self-administration neuronal ensemble and then ablated these neurons using postsession Daun02 injections. We tested the hypothesis that ablating the cocaine self-administration ensemble during induction day would lead to decreased cocaine seeking without influencing food seeking during the test day. The design of experiment 3 is shown in Figure $3 \mathrm{~A}$.

\subsection{1 | Training and induction day}

Figure 3B shows the mean \pm SEM number of active and inactive lever presses during the self-administration phase, and Figure S3-1C shows the results of the weekly choice sessions. As in experiments 1 and 2 , the rats showed reliable cocaine self-administration with higher responding for the food reward (Figure $3 \mathrm{~B}$ ) and higher preference for the food reward during the choice sessions (Figure S3-1C).

\subsection{2 | Induction day (Figure 3D)}

On induction day, nonreinforced lever pressing for cues associated with cocaine seeking was assessed for $30 \mathrm{~min}$. We then placed rats in their home cages for 60 min and injected Daun02 or vehicle into vmPFC. There were no group differences in lever presses prior to vehicle or Daun02 injections between the groups (Figure 3D; $p>0.1$ ).

\subsection{3 | Reward-seeking test (Figure 3E)}

We found no significant differences in behavioral responses between male and female rats on test day (Figure S3) and therefore combined these groups for analysis. Daun02 injections decreased cocaine seeking but increased food seeking during testing. The two-factorial mixed ANCOVA comparing active lever presses, which included the between-subjects factors of Daun02 condition, the within-subjects factors of Reward type, and the covariate Inactive lever presses, showed significant effects of Reward type $\left(F_{1,25}=17.5, p<0.0001\right)$ and an interaction between Daun02 condition $\times$ Reward type $\left(F_{1,25}=8.0, p=0.009\right)$ but no main effect of Daun02 $\left(F_{1,25}=0.2\right.$, $p=0.7)$. Post-hoc analysis revealed that Daun02 inactivation of cocaine-paired ensembles decreased cocaine-paired lever presses ( $p=0.042$ ) but increased food-paired lever presses $(p=0.043)$. These results support our hypothesis that selective ablation of the putative 
cocaine-seeking ensemble during induction day would selectively decrease cocaine but not food seeking on test day.

\section{4 | DISCUSSION}

We used alternating food versus cocaine self-administration training sessions and the Daun02 inactivation procedure to test the specificity of neuronal ensembles in vmPFC in mediating food and cocaine seeking. We found that Daun02 inactivation of the neuronal ensemble associated with recall of food self-administration did not influence subsequent food or cocaine seeking. In contrast, selective ablation of the cocaine-associated neuronal ensemble decreased subsequent cocaine seeking and increased food seeking. These results are consistent with our previous findings showing that inactivation of neuronal ensembles associated with recall of cocaine self-administration decreases cocaine seeking. ${ }^{24}$ Because Daun02 interacts exclusively with $\beta$-gal in Fos-expressing ensembles, ${ }^{16}$ the results of specific modulation of cocaine seeking after Daun02 injections suggest that a distinct neuronal ensemble within vmPFC encodes the cocaine selfadministration memory that appears functionally separable from the ensemble activated by the food self-administration memory.

\subsection{Role of vmPFC neuronal ensembles in cocaine and food seeking}

In the present study, we tested the specificity of neuronal ensembles in encoding natural versus drug rewards using the Daun02 inactivation procedure ${ }^{28}$ to selectively inactivate either the food- or cocaineseeking neuronal ensemble. Our results expand on our recent findings showing that neuronal ensembles in vmPFC are critical for food seeking ${ }^{27}$ and cocaine seeking. ${ }^{24}$ In these previous studies, we found that the infralimbic cortex is involved in both promoting and repressing seeking for food or cocaine rewards, which suggests that the popular hypothesis ${ }^{33,34}$ (based on nonspecific whole brain region inactivation) that infralimbic cortex only represses whereas prelimbic cortex only promotes reward seeking should be revised. The previous Daun02 studies $^{19-21,24,27,35}$ used the same volume and injections of Daun02 into vmPFC and found that the mPFC region affected by Daun02 ablation encodes memories underlying both repression and promoting reward seeking in the same brain area. The current results indicate that the neuronal ensemble encoding the cocaine-seeking behavior is specific to the cocaine memory, because rats selectively decreased cocaine seeking on test day. The increased food-seeking behavior on test day in those same rats after inactivation of the cocaine-seeking ensemble on induction day suggests that the mechanism underlying food and cocaine seeking is distinct. On the other hand, ablating the food-seeking ensemble on induction day did not significantly alter either food or cocaine seeking on test day. This may be due in part to redundancy with ensembles in other brain regions being sufficient for recall of the food self-administration memory. This is likely true for the cocaine self-administration memory as well, but vmPFC may play a more significant role in cocaine-associated memories than in foodassociated memories. Nevertheless, our results suggest that controlling drug-associated versus food-associated (or other nondrug rewards) learned associations may be possible through manipulations of neuronal ensembles. The targeting of these small, sparsely distributed subgroups of neurons could yield long-term decreases of drug seeking selectively while maintaining other nondrug reward-seeking behaviors intact.

Single-unit recordings of mPFC neurons during operant responding have found that different rewards or aspects of rewards cause firing in different neurons. ${ }^{36-38}$ Neurons activated by one reinforcer (cocaine) are often not activated by a second reinforcer (heroin). ${ }^{36}$ This suggests that the two learned associations activate largely different populations of neurons, likely different neuronal ensembles. The Daun02 inactivation experiments presented here build upon this literature and suggest that separate populations of neurons are not only activated by distinct rewarding stimuli but can also be distinguished by whether they play a functional role in cocaine-seeking or not. The present results also mirror findings showing distinct roles of neuronal ensembles in mediating diverse behaviors. We and others found that different neuronal ensembles in the vmPFC mediate selfadministration versus extinction of food and cocaine seeking. ${ }^{23,26,27}$ In a separate study, we found that neuronal ensembles in dorsomedial striatum are critical for methamphetamine seeking but not food seeking. ${ }^{19}$ Unexpectedly, we found that inactivation of the cocaine-paired ensemble on induction day increased subsequent food seeking on the test day. Because the excitability of relevant neuronal ensembles is actively increased and the excitability of nonensemble neurons is actively decreased, ${ }^{35}$ it is possible that inactivation of the cocaineseeking ensemble may release the food-seeking ensemble from inhibition, thereby increasing food-seeking behavior.

The current study has some methodological and interpretation limitations that we discuss below. First, although the timing of Daun02 injections is designed to capture neuronal ensembles that are activated following exposure to learned cues in a discrete brain area, multiple unrelated ensembles can also be affected by these injections. One possible set of ensembles may encode prediction error detection and extinction learning during induction day, when the reinforcers are withheld. Another set of neuronal ensembles may be responsible for discriminating between active and inactive levers for the alternate reinforcer or suppressing pressing on the nonreinforced lever. Also, the use of a shared inactive lever in close proximity to the food-paired lever that was always present in the operant chamber may have made the memories for food versus cocaine self-administration less distinct. Perhaps providing more distinction between the cocaine and food self-administration conditions by removing the inactive lever or adding a second inactive lever near the cocaine-paired lever would have resulted in more distinct associations and thus more distinct ensembles. Nevertheless, despite the use of a shared inactive lever, we find a significant decrease in cocaine but not food seeking after inactivation of the cocaine-paired ensemble, suggesting that the two ensembles are distinct. This interpretation presumes that inactivating extraneous ensembles would not influence reward-seeking behavior 
for the alternative reward. It is plausible that we are inactivating peripheral neuronal ensembles that can impact seeking for the alternative reward, driving the increase in food seeking.

Lastly, Daun02 ablation in Fos-LacZ rats is typically confirmed by measuring $\beta$-galactosidase labeling after ensemble reactivation during the test day to show decreased $\beta$-galactosidase expression in the region of interest. In this experiment, we opted for a within-subjects testing approach to minimize the number of rats required, which made such a $\beta$-galactosidase test uninterpretable. Additionally, although we limited our surgical placement to a relatively narrow region within the vmPFC to minimize off-target effects, we cannot eliminate the possibility that Daun02 reached regions outside of the vmPFC, such as diffusion up the cannula shaft to dorsal mPFC.

\section{5 | CONCLUSION}

Inactivation of neuronal ensembles in vmPFC associated with recall of cocaine self-administration specifically decreases cocaine seeking but not food seeking. In contrast, inactivation of neuronal ensembles encoding food self-administration did not influence food or cocaine seeking. These data suggest that the neuronal ensemble activated by cocaine seeking in the vmPFC is functionally separate from the ensemble activated by food seeking.

\section{ACKNOWLEDGMENTS}

This research was supported by the National Institute on Drug Abuse, Intramural Research Program, NIH. BLW was supported by a grant from the National Institute on Drug Abuse (NIDA) (grant no. 4ROODA042102-02) and a NARSAD Young Investigator Grant from the Brain and Behavior Research Foundation.

\section{CONFLICT OF INTEREST}

The authors declare no competing financial interests.

\section{AUTHORS CONTRIBUTIONS}

$\mathrm{BW}, \mathrm{LK}, \mathrm{BH}, \mathrm{DC}$, and YS designed the behavioral experiments. LK, $\mathrm{BW}, \mathrm{MV}, \mathrm{RM}$, and JMB performed surgeries or intracranial infusions on the rats. $\mathrm{LK}, \mathrm{RQF}$, and $\mathrm{BW}$ were responsible for collecting behavioral and histochemical data. LK and BW wrote the manuscript. All authors provided critical reviews of the content and approved the final version for publication.

\section{ORCID}

Marco Venniro (D) https://orcid.org/0000-0001-7653-957X Richard Quintana-Feliciano (D) https://orcid.org/0000-0002-58354683

Rajtarun Madangopal (D) https://orcid.org/0000-0001-6202-302X Jennifer M. Bossert (iD https://orcid.org/0000-0001-5636-4071 Yavin Shaham (D) https://orcid.org/0000-0002-8242-3319 Bruce T. Hope (D) https://orcid.org/0000-0001-5804-7061 Brandon L. Warren (D) https://orcid.org/0000-0002-5922-9382

\section{REFERENCES}

1. Washton AM, Tatarsky A. Adverse effects of cocaine abuse. NIDA Res Monogr. 1984;49:247-254.

2. Cregler LL. Adverse health consequences of cocaine abuse. J Natl Med Assoc. 1989;81(1):27-38.

3. Mateu-Gelabert P, Maslow C, Flom PL, Sandoval M, Bolyard M, Friedman SR. Keeping it together: stigma, response, and perception of risk in relationships between drug injectors and crack smokers, and other community residents. AIDS Care. 2005;17(7):802-813.

4. Childress AR, McLellan AT, Ehrman R, O'Brien CP. Classically conditioned responses in opioid and cocaine dependence: a role in relapse? NIDA Res Monogr. 1988;84:25-43.

5. Childress A, Ehrman R, McLellan AT, O'Brien C. Conditioned craving and arousal in cocaine addiction: a preliminary report. NIDA Res Monogr. 1988;81:74-80.

6. Kufahl PR, Li Z, Risinger RC, et al. Neural responses to acute cocaine administration in the human brain detected by fMRI. Neuroimage. 2005;28(4):904-914

7. Garavan H, Pankiewicz J, Bloom A, et al. Cue-induced cocaine craving: neuroanatomical specificity for drug users and drug stimuli. Am J Psychiatry. 2000;157(11):1789-1798.

8. Ciccocioppo R, Sanna PP, Weiss F. Cocaine-predictive stimulus induces drug-seeking behavior and neural activation in limbic brain regions after multiple months of abstinence: reversal by $D(1)$ antagonists. Proc Natl Acad Sci U S A. 2001;98(4):1976-1981.

9. Gao P, Limpens JH, Spijker S, Vanderschuren L, Voorn P. Stable immediate early gene expression patterns in medial prefrontal cortex and striatum after long-term cocaine self-administration. Addict Biol. 2017;22(2):354-368.

10. Robinson TE, Gorny G, Mitton E, Kolb B. Cocaine self-administration alters the morphology of dendrites and dendritic spines in the nucleus accumbens and neocortex. Synapse. 2001;39(3):257-266.

11. Robinson TE, Kolb B. Alterations in the morphology of dendrites and dendritic spines in the nucleus accumbens and prefrontal cortex following repeated treatment with amphetamine or cocaine. Eur J Neurosci. 1999;11(5):1598-1604.

12. Otis JM, Mueller D. Inhibition of beta-adrenergic receptors induces a persistent deficit in retrieval of a cocaine-associated memory providing protection against reinstatement. Neuropsychopharmacology. 2011;36(9):1912-1920.

13. Otis JM, Namboodiri VM, Matan AM, et al. Prefrontal cortex output circuits guide reward seeking through divergent cue encoding. Nature. 2017;543(7643):103-107.

14. Pennartz CM, Groenewegen HJ, Lopes da Silva FH. The nucleus accumbens as a complex of functionally distinct neuronal ensembles: an integration of behavioural, electrophysiological and anatomical data. Prog Neurobiol. 1994;42(6):719-761.

15. Guzowski JF, Knierim JJ, Moser El. Ensemble dynamics of hippocampal regions CA3 and CA1. Neuron. 2004;44(4):581-584.

16. Cruz FC, Koya E, Guez-Barber DH, et al. New technologies for examining the role of neuronal ensembles in drug addiction and fear. Nat Rev Neurosci. 2013;14(11):743-754.

17. Wilson MA, McNaughton BL. Dynamics of the hippocampal ensemble code for space. Science. 1993;261(5124):1055-1058.

18. Bossert JM, Stern AL, Theberge FR, et al. Ventral medial prefrontal cortex neuronal ensembles mediate context-induced relapse to heroin. Nat Neurosci. 2011;14(4):420-422.

19. Caprioli D, Venniro M, Zhang M, et al. Role of dorsomedial striatum neuronal ensembles in incubation of methamphetamine craving after voluntary abstinence. J Neurosci. 2017;37(4):1014-1027.

20. Cruz FC, Babin KR, Leao RM, et al. Role of nucleus accumbens shell neuronal ensembles in context-induced reinstatement of cocaineseeking. J Neurosci. 2014;34(22):7437-7446. 
21. Fanous S, Goldart EM, Theberge FR, Bossert JM, Shaham Y, Hope BT. Role of orbitofrontal cortex neuronal ensembles in the expression of incubation of heroin craving. J Neurosci. 2012;32(34):11600-11609.

22. Pfarr S, Meinhardt MW, Klee ML, et al. Losing control: excessive alcohol seeking after selective inactivation of cue-responsive neurons in the infralimbic cortex. J Neurosci. 2015;35(30):10750-10761.

23. Suto N, Laque A, De Ness GL, et al. Distinct memory engrams in the infralimbic cortex of rats control opposing environmental actions on a learned behavior. Elife. 2016;5:e21920, 1-12.

24. Warren BL, Kane L, Venniro M, et al. Separate vmPFC ensembles control cocaine self-administration versus extinction in rats. J Neurosci. 2019;39(37):7394-7407.

25. Funk D, Coen K, Tamadon S, Hope BT, Shaham Y, Le AD. Role of central amygdala neuronal ensembles in incubation of nicotine craving. J Neurosci. 2016;36(33):8612-8623.

26. Laque A, De Ness GL, Wagner GE, et al. Anti-relapse neurons in the infralimbic cortex of rats drive relapse-suppression by drug omission cues. Nat Commun. 2019;10:a3934, 1-15.

27. Warren BL, Mendoza MP, Cruz FC, et al. Distinct Fos-expressing neuronal ensembles in the ventromedial prefrontal cortex mediate food reward and extinction memories. J Neurosci. 2016;36(25):6691-6703.

28. Koya E, Golden SA, Harvey BK, et al. Targeted disruption of cocaineactivated nucleus accumbens neurons prevents context-specific sensitization. Nat Neurosci. 2009;12(8):1069-1073.

29. Rubio FJ, Liu QR, Li X, et al. Context-induced reinstatement of methamphetamine seeking is associated with unique molecular alterations in Fos-expressing dorsolateral striatum neurons. J Neurosci. 2015;35 (14):5625-5639.

30. Caprioli D, Venniro M, Zeric T, et al. Effect of the novel positive allosteric modulator of metabotropic glutamate receptor 2 AZD8529 on incubation of methamphetamine craving after prolonged voluntary abstinence in a rat model. Biol Psychiatry. 2015;78(7):463-473.

31. Lenoir M, Serre F, Cantin L, Ahmed SH. Intense sweetness surpasses cocaine reward. PLoS ONE. 2007;2(8):e698, 1-10.

32. Madsen $\mathrm{HB}$, Ahmed $\mathrm{SH}$. Drug versus sweet reward: greater attraction to and preference for sweet versus drug cues. Addict Biol. 2015;20(3): 433-444.
33. Peters J, Kalivas PW, Quirk GJ. Extinction circuits for fear and addiction overlap in prefrontal cortex. Learn Mem. 2009;16(5):279-288.

34. Peters J, Vallone J, Laurendi K, Kalivas PW. Opposing roles for the ventral prefrontal cortex and the basolateral amygdala on the spontaneous recovery of cocaine-seeking in rats. Psychopharmacology (Berl). 2008;197(2):319-326.

35. Whitaker LR, Warren BL, Venniro M, et al. Bidirectional modulation of intrinsic excitability in rat prelimbic cortex neuronal ensembles and non-ensembles after operant learning. J Neurosci. 2017;37(36):88458856.

36. Chang JY, Janak PH, Woodward DJ. Comparison of mesocorticolimbic neuronal responses during cocaine and heroin self-administration in freely moving rats. J Neurosci. 1998;18(8):3098-3115.

37. West EA, Saddoris MP, Kerfoot EC, Carelli RM. Prelimbic and infralimbic cortical regions differentially encode cocaine-associated stimuli and cocaine-seeking before and following abstinence. Eur $J$ Neurosci. 2014;39(11):1891-1902.

38. Hyman JM, Ma L, Balaguer-Ballester E, Durstewitz D, Seamans JK. Contextual encoding by ensembles of medial prefrontal cortex neurons. Proc Natl Acad Sci U S A. 2012;109(13):5086-5091.

\section{SUPPORTING INFORMATION}

Additional supporting information may be found online in the Supporting Information section at the end of this article.

How to cite this article: Kane L, Venniro M, Quintana-

Feliciano R, et al. Fos-expressing neuronal ensemble in rat ventromedial prefrontal cortex encodes cocaine seeking but not food seeking in rats. Addiction Biology. 2020;e12943. https://doi.org/10.1111/adb.12943 